\title{
RESIDUE THEOREMS OF HARMONIC FUNCTIONS OF THREE VARIABLES
}

\author{
STEFAN BERGMAN
}

1. Introduction. The connection between analytic functions of one complex variable and harmonic functions of two real variables is used in the study of the latter functions. The analogy suggests generalizations of these methods of attack to the case of three variables.

Suppose that $O$ is some operation which transforms the class of analytic functions of the complex variable $u$ into the class of harmonic functions of three variables $x_{1}, x_{2}, x_{3}$. If $O$ possesses the property that to every harmonic polynomial of the $n$th degree there corresponds an expression of the form $A\left[u\left(x_{1}, x_{2}, x_{3}\right)\right]^{n}$, where $u$ is a linear expression in $x_{1}, x_{2}, x_{3}$, then such an operation yields a representation of spherical harmonics.

The existing representations of the spherical harmonics lead us to several operations $\boldsymbol{O}$. The operation $\mathrm{P}$ described below (a generalization of the known Whittaker's formula) seems to be the most suitable for the questions considered in this note.

Let $F$ be the class of analytic functions of complex variables $u$ and $\zeta$, where $u=x_{1}+(i / 2)\left(x_{2}+i x_{3}\right) \zeta^{-1}+(i / 2)\left(x_{2}-i x_{3}\right) \zeta$. By the operation

$$
\mathrm{P}\left(f, \mathscr{Q}^{1}, \mathfrak{I}\right)=(1 / 2 \pi i) \int_{2^{1}} f(u, \zeta) d \zeta, \quad \mathfrak{X}=\left(x_{1}, x_{2}, x_{3}\right) \in \mathcal{U}^{3}(\mathfrak{T})
$$

$F$ is transformed into the class of (complex) harmonic ${ }^{1}$ functions (see $\left.[1,2]^{2}\right) . V^{3}(\mathfrak{T})$ denotes a sufficiently small neighborhood of the point $\mathfrak{I}=\left(t_{1}, t_{2}, t_{3}\right)$, and $2^{1}$ is a closed curve in the $\zeta$-plane.

$\mathbf{P}\left(f, 2^{1}, \mathfrak{I}\right)$ is a functional. If we carry out the integration for $\mathfrak{X} \in \mathcal{U}^{\mathfrak{3}}(\mathfrak{I})$ and we continue analytically the obtained harmonic function $H(\mathfrak{X})$ to a point $\mathfrak{Y} \in \mathcal{U}^{3}(\mathfrak{B})$, then the continued function is not necessarily equal to $\mathbf{P}\left(f, \mathscr{Q}^{1}, \mathfrak{B}\right)$. This is the reason why it is necessary to indicate the point $\mathfrak{I}$ in the neighborhood of which the integration is carried out.

Presented to the Society, April 3, 1942 under the title Three-dimensional flow of a perfect incompressible fluid and its singularities; received by the editors April 13, 1942.

${ }^{1}$ Here and hereafter harmonic functions mean harmonic functions of three real variables $x_{1}, x_{2}, x_{3}$, except when we specifically state that we mean harmonic functions of two variables. By taking the real or imaginary part in the formulas for complex harmonic functions we obtain analogous results for real harmonic functions.

${ }^{2}$ The numbers in brackets refer to the bibliography.

$E[\cdots]$ means the set of points satisfying the relations indicated in the brackets. Superscripts over a manifold indicate its dimensionality. 
The analogy suggests further consideration of harmonic vectors $\mathfrak{S}(\mathfrak{X})=H^{(1)}(\mathfrak{X}) \mathfrak{i}_{1}+H^{(2)}(\mathfrak{X}) \mathfrak{i}_{2}+H^{(3)}(\mathfrak{X}) \mathfrak{i}_{3}$, in addition to a single function, where $H^{(k)}$ are harmonic functions connected by certain differential relations. ${ }^{3} \mathfrak{i}_{k}$ is the unit vector in the $x_{k}$ direction.

In $\$ \S 2$ and 3 we shall consider harmonic vectors

$$
\begin{aligned}
\mathfrak{S}(\mathfrak{X})=\mathfrak{P}\left(f, 2^{1}, \mathfrak{I}\right) \equiv & \mathrm{P}\left[f, 2^{1}, \mathfrak{T}\right] \mathrm{i}_{1}+\mathrm{P}\left[(i / 2)\left(\zeta+\zeta^{-1}\right) f, 2^{1}, \mathfrak{T}\right] \mathfrak{i}_{2} \\
& +\mathrm{P}\left[(1 / 2)\left(\zeta-\zeta^{-1}\right) f, 2^{1}, \mathfrak{T}\right] \mathfrak{i}_{3} .
\end{aligned}
$$

Clearly, curl $\mathfrak{S}=0, \operatorname{div} \mathfrak{S}=0$ at every point at which all components of $\mathfrak{S}$ are regular.

Consider vectors $\mathfrak{S}(\mathfrak{Y})=H^{(1)}(\mathfrak{Y}) \mathfrak{i}_{1}+H^{(2)}(\mathfrak{Y}) \mathfrak{i}_{2}, \mathfrak{Y}=\left(y_{1}, y_{2}\right)$ of two real variables $y_{1}, y_{2}$ for which we have $\operatorname{curl} \mathfrak{S}=0, \operatorname{div} \mathfrak{S}=0$. $\int_{\mathfrak{J}^{1}} \mathfrak{S} \cdot d \mathfrak{V}=0$ if $J^{1}$ is a closed curve, and if $J^{1}$ can be shrunk to a point in a regularity domain of $\mathfrak{S}$. ( $\cdot$ means the scalar product.) If singularities of $\mathfrak{S}$ lie inside of $\mathfrak{J}^{1}$ or $\mathfrak{S}$ is a many valued function the residue theorem yields well known relations.

Let $R^{2}$ be some simply connected domain in which $\Im^{1}$ lies, and let $\mathfrak{S}=\mathfrak{S}_{1}+\sum_{k=1}^{n} \mathfrak{S}_{k}$, where $\mathfrak{S}_{1}$ is a vector which is regular in $\mathbb{R}^{2}$. Then

$$
\int_{\mathfrak{J}^{1}} \mathfrak{S} \cdot d \mathfrak{Y}=\int_{\mathfrak{J}^{1}} \mathfrak{S}_{1} \cdot d \mathfrak{Y}+\sum_{k=1}^{n} \int_{\mathfrak{J}^{1}} \mathfrak{S}_{k} \cdot d \mathfrak{Y} \text {. }
$$

In this connection we make the trivial simplification that we limit ourselves to the case of suitable standard vectors $\subseteq$ which possess one singular point (or one connected singular line in the case of three variables).

If $\mathfrak{S}(\mathfrak{Y})=\operatorname{Im}\left[(z-\alpha)^{-1}\right] \mathfrak{i}_{1}+\operatorname{Re}\left[(z-\alpha)^{-1}\right] \mathfrak{i}_{2}$ then $\int_{\mathfrak{J}^{1}} \mathfrak{S}(\mathfrak{Y}) \cdot d \mathfrak{Y}=2 \pi$ if $\alpha$ lies inside of $\mathfrak{J}^{1}$. Since $\subseteq$ can be considered as a harmonic vector in three variables the above relation may be formulated in the following form. Let $\mathfrak{S}(\mathfrak{X})$ be a harmonic vector which in appropriate coordinates $y_{1}, y_{2}, y_{3}$ may be written

$$
\widetilde{S}=\operatorname{Im}\left(y_{1}+i y_{2}-\alpha\right)^{-1} \mathfrak{i}_{1}+\operatorname{Re}\left(y_{1}+i y_{2}-\alpha\right)^{-1} \mathfrak{i}_{2}+0 \cdot \mathfrak{t}_{3} .
$$

Then $\int_{\mathfrak{I}^{1}} \mathfrak{S}(\mathfrak{X}) \cdot d \mathfrak{X}=2 \pi$ if $\mathfrak{J}^{1}$ cannot be reduced to a point without cutting $\mathrm{E}\left[y_{3}=0, y_{1}+i y_{2}=\alpha\right]$. As it is known (see $[1,2]$ ) there exist algebraic harmonic functions with singularity lines or points [see

${ }^{3}$ Harmonic vectors are met in physical applications especially in the theory of a perfect incompressible fluid.

The above mentioned differential relations can vary in nature depending upon the question which has to be considered. We limit ourselves in this paper to special relations, namely, curl $\mathfrak{S}=0, \operatorname{div} \mathfrak{S}=0$ in $\S \S 2$ and 3 and (4.3) in $\$ 4$. However, in many instances with certain changes we may extend our results to much more general relations. 
(2.5) p. 166] which cannot be represented as harmonic functions of two variables. In $\S \S 2$ and 3 we in troduce harmonic vectors $\mathfrak{S}(\mathfrak{X})$ with such singularities and consider

$$
\sum_{k} \int_{\mathfrak{X}_{k}^{(1)}}^{\mathfrak{X}_{k}^{(2)}} \Im_{k}(\mathfrak{X}) \cdot d \mathfrak{X} .
$$

Here $\mathfrak{S}_{k}$ are branches of $\subseteq$ and $\mathfrak{X}_{k}^{(\mathrm{H})}$ points which satisfy certain algebraic relations. We show that the above expression is equal to a quantity connected with the singularity, which may be interpreted as the residue of S. $^{4}$

A theorem of related character concerning surface integrals is given in $\$ 4$.

As pointed out before, the operation $\mathbf{P}$ transforms a polynomial in $u$ and $\zeta$ into a harmonic polynomial of the same degree.

As we shall see below a rational function $f$ is in general transformed into an algebraic harmonic function of $x_{1}, x_{2}, x_{3}$. The question arises whether every algebraic harmonic function $A\left(x_{1}, x_{2}, x_{3}\right)$ can be represented in the form $\mathbf{P}\left(f, 2^{1}, \mathfrak{I}\right)$ where $f$ is a rational function in $u$ and $\zeta$. This question has not been answered. However, from the result of [2, p. 637] follows that $A\left(x_{1}, x_{2}, x_{3}\right)$ can be represented in the above form with $f=\int_{0}^{1} a(u, \zeta, T) d T, a$ being an algebraic function in $u, \zeta, T$.

2. Singularities of harmonic functions. The introduction of the operator (1.1) gives a simple procedure for the construction of standard functions with singularities. Suppose that $f(u, \zeta)$ has poles, say for instance $f=p / q$, where $p$ and $q$ are polynomials in $u$ and $\zeta$. For simplicity's sake we shall suppose that $q=[u-\phi(\zeta)] \zeta^{s}$ where $\phi(\zeta)$ is a polynomial in $\zeta$ and $s$ a non-negative integer.

In order to study the functional

$$
\mathrm{P}\left[p / q, 2^{1}, \mathfrak{T}\right]=\frac{1}{2 \pi i} \int_{\mathscr{2}^{1}} \frac{p(u, \zeta)}{q(u, \zeta)} d \zeta, \mathfrak{X} \in \mathcal{V}^{3}(\mathfrak{T}), \mathfrak{X}=\left(x_{1}, x_{2}, x_{3}\right),
$$

as a function of $\mathfrak{T}$ it is useful to consider (2.1) in the (five-dimensional) space $x_{1}, x_{2}, x_{3}, \xi, \eta$; where $\zeta=\xi+i \eta$.

$$
Z^{3}=\mathrm{E}\left\{q\left[\left(x_{1}+(i / 2)\left(x_{2}+i x_{3}\right) \zeta^{-1}+(i / 2)\left(x_{2}-i x_{3}\right) \zeta\right), \zeta\right]=0\right\}
$$

is the singularity manifold of $p / q . Z^{3}$ can also be written in the form

$$
Z^{3}=\mathrm{E}\left[\zeta=\zeta_{\nu}(\mathfrak{X}), \nu=1,2, \cdots, n\right]
$$

where $\zeta_{\nu}$ are algebraic functions of $x_{1}, x_{2}, x_{3}$ and $n$ the degree of $q$ in $\zeta$.

4 This result has a certain similarity with Abel's theorem in the theory of integrals of algebraic functions. 
At every point $\mathfrak{X}$, except those which lie in

$$
\mho^{1}=\mathrm{E}\left\{\prod_{k \neq s}\left[\zeta_{s}(\mathfrak{X})-\zeta_{k}(\mathfrak{X})\right]^{2}=0\right\}
$$

we have $n$ distinct branches $Z_{\nu}^{3}=\mathrm{E}\left[\zeta=\zeta_{\nu}(\mathfrak{X})\right], \nu=1,2, \cdots, n$ of $Z^{3}$.

Suppose $\mathfrak{D} \in \mathcal{U}^{1}$, and $\mathcal{C}_{\nu}^{1}, \nu=1,2, \cdots, n$, are chosen in such a way that one and only one $\zeta_{\nu}(\mathfrak{D})$ lies inside of each $\mathcal{C}_{\nu}^{\mathbf{1}}$. Then by the residue theorem $\mathrm{P}\left[p / q, C_{\nu}^{1}, \mathfrak{D}\right]=S_{\nu}(\mathfrak{X})$, where $S_{\nu}$ is the corresponding branch of

$$
\begin{gathered}
S(\mathfrak{X})=\frac{p\left[\left(x_{1}+(i / 2)\left(x_{2}+i x_{3}\right) \zeta^{-1}+(i / 2)\left(x_{2}-i x_{3}\right) \zeta\right), \zeta\right]}{\partial\left\{q\left[\left(x_{1}+(i / 2)\left(x_{2}+i x_{3}\right) \zeta^{-1}+(i / 2)\left(x_{2}-i x_{3}\right) \zeta\right), \zeta\right]\right\} / \partial \zeta} \\
q\left[\left(x_{1}+(i / 2)\left(x_{2}+i x_{3}\right) \zeta^{-1}+(i / 2)\left(x_{2}-i x_{3}\right) \zeta\right), \zeta\right]=0 .
\end{gathered}
$$

$S(\mathfrak{X})$ is an algebraic function which possesses $n$ branches. We denote the (multiply covered) $x_{1}, x_{2}, x_{3}$-space in which $S(\mathfrak{X})$ is univalent by $R^{3}$; its sheets (which are supposed to be obtained by appropriate cuts), by $R_{\nu}^{3}$. The singularity line of $S(\mathfrak{X})$ is

$$
\begin{aligned}
q\left[\left(x_{1}+(i / 2)\left(x_{2}+i x_{3}\right) \zeta^{-1}+(i / 2)\left(x_{2}-i x_{3}\right) \zeta\right), \zeta\right] & =0 \\
\frac{\partial q\left[\left(x_{1}+(i / 2)\left(x_{2}+i x_{3}\right) \zeta^{-1}+(i / 2)\left(x_{2}-i x_{3}\right) \zeta\right), \zeta\right]}{\partial \zeta} & =0 .
\end{aligned}
$$

ExAMPLE. $\mathrm{P}\left[(u-a i)^{-1} \zeta^{-1}, 2^{1}, \mathfrak{T}\right], 2^{1}=\mathrm{E}\left[\zeta=e^{i t}, 0 \leqq t \leqq 2 \pi\right], a \geqq 0$, $t_{1} \geqq 0$ is a two valued function. (See $[1$, p. 655].) If $a>0$ then the singularity line $\mathrm{E}\left[x_{1}=0, x_{2}^{2}+x_{3}^{2}=a^{2}\right]$ is the branch line. For $a=0$ this branch line shrinks to a point, both branches $S_{k}(\mathfrak{X})=(-1)^{k+1} / r$ for $k=1,2, r=\left(x_{1}^{2}+x_{2}^{2}+x_{3}^{2}\right)^{1 / 2}$, are connected only at the origin.

We further note that $\mathrm{P}\left[(1 / 2)\left(\zeta+\zeta^{-1}\right) u^{-1} \zeta^{-1}, \mathcal{Q}^{1}, \mathfrak{T}\right]=-i r^{-1} \cos \phi$ $\cdot \tan (\theta / 2), \quad \mathrm{P}\left[(1 / 2 i)\left(\zeta-\zeta^{-1}\right) u^{-1} \zeta^{-1}, 2^{1}, \mathfrak{T}\right]=-i r^{-1} \sin \phi \tan (\theta / 2)$ for $t_{1}>0$. Here $r, \theta, \phi$ are polar coordinates.

In study of the representation (2.1) the points $\mathfrak{X}$ which satisfy the equation $q\left[\left(x_{1}+(i / 2)\left(x_{2}+i x_{3}\right) \zeta^{-1}+(i / 2)\left(x_{2}-i x_{3}\right) \zeta\right), \zeta\right]=0$ (for an appropriate $\zeta)$ are of importance. In this connection it is useful to introduce certain curves, $\mathcal{L}^{1}(\zeta)$.

${ }^{5}$ We suppose here that $\zeta=0$ lies outside of $\mathcal{C}_{\nu}^{1}$. Otherwise, and also for the evaluation of (2.1) if the degree of $q$ (in $u$ ) is higher than 1, see [2, pp. 644-650], especially the formulas (7) and (10).

We note that the $\mathbb{R}_{\nu}^{3}$ are not necessarily connected with each other in the real. $x_{1}, x_{2}, x_{3}$-space. 
By the correspondence

$$
\mathfrak{X} \rightarrow u=x_{1}+(i / 2)\left(x_{2}+i x_{3}\right) \zeta^{-1}+(i / 2)\left(x_{2}-i x_{3}\right) \zeta
$$

we associate to every point $\mathfrak{X}$ of the $x_{1}, x_{2}, x_{3}$-space a point $u$ of the complex plane. On the other hand to the point $A+i B$ of the $u$-plane there corresponds a line $\mathcal{P}^{1}(\zeta, A+i B)$ in the $x_{1}, x_{2}, x_{3}$-space. If we write $x_{2}+i x_{3}=\rho e^{i \varphi}, \zeta=\tau e^{i \alpha}$ then from $u=A+i B$ it follows that $i e^{i(\varphi-\alpha)} \tau^{-1}+2 x_{1} \rho^{-1}+i e^{-i(\varphi-\alpha)} \tau=2 \rho^{-1}(A+i B)$ or

$$
\mathbb{P}^{1}\left(\tau e^{i \alpha}, A+i B\right)=\mathrm{E}\left[\rho^{2}=4\left(\frac{B}{\tau+\tau^{-1}}\right)^{2}+4\left(\frac{A-x_{1}}{\tau-\tau^{-1}}\right)^{2},\right.
$$

We put

$$
\left.\varphi=\alpha+\operatorname{arc} \cos \frac{2 B \rho^{-1}}{\tau+\tau^{-1}}\right] \text {. }
$$

$$
\mathcal{L}^{1}(\zeta)=\mathscr{P}^{1}[\zeta, \phi(\zeta)]
$$

where $\phi(\zeta)$ is the polynomial introduced on p. 165 . For $\mathfrak{X} \in \mathcal{L}^{1}\left(\zeta_{0}\right)$ we have

$$
\begin{aligned}
q\left(u, \zeta_{0}\right) & =q\left[\left(x_{1}+(i / 2)\left(x_{2}+i x_{3}\right) \zeta_{0}^{-1}+(i / 2)\left(x_{2}-i x_{3}\right) \zeta_{0}\right), \zeta_{0}\right] \\
& =\zeta_{0}^{8}\left[u_{0}-\phi\left(\zeta_{0}\right)\right]=0
\end{aligned}
$$

therefore

$$
\mathcal{L}^{1}\left(\zeta_{0}\right)=Z^{3} \cap \mathrm{E}\left(\zeta=\zeta_{0}\right)
$$

Since in $R_{1}^{3}, Z^{3}=S_{p=1}^{n} Z_{\nu}^{3}$, we can write $\mathcal{L}^{1}(\zeta)=S_{\nu=1}^{n} \mathcal{L}_{\nu}^{1}(\zeta)$ where $\mathcal{L}_{\nu}^{1}\left(\zeta_{0}\right)=Z_{\nu}^{3} \cap \mathrm{E}\left[\zeta=\zeta_{0}\right]$

Let $2^{1}$ be a closed curve in the $\zeta$-plane. We suppose (for simplicity's sake) that one and only one point $\zeta_{k}(\mathfrak{D})$, say $\zeta_{1}(\mathfrak{D})$, lies inside of ${ }^{1}$. Then $\mathrm{P}\left[p / q, 2^{1}, \mathfrak{I}\right]=S_{1}(\mathfrak{X})$ for $\mathfrak{I}=\mathfrak{D}$. However, if $\mathfrak{I}$ varies in $\mathbb{R}_{1}^{3}$ new branches $Z_{\nu}^{3}$ can slip in or out from $2^{1}$. Since $Z^{3}$ is a continuous manifold, $Z_{\nu}^{3}$ can enter $2^{1}$ only for such values of $\mathfrak{X}$ for which $Z_{\nu}^{3}$ has intersection with $\mathrm{E}\left[\zeta \in 2^{1}\right]$, that is for $\mathfrak{X} \in Z_{\nu}^{3} \cap \mathrm{E}\left[\zeta \in 2^{1}\right]=S_{\zeta \in \mathcal{Q}^{1} \mathcal{L}_{\nu}^{1}(\zeta)}$ $=\mathcal{X}_{\nu}^{2}\left(2^{1}\right)$. Thus, if the point $\mathfrak{I}$ in the operator $(2.1)$ moves and passes through $\mathscr{X}^{2}\left(2^{1}\right)$, the integral $\mathrm{P}\left[p / q, 2^{1}, \mathfrak{T}\right]$ has a jump, ${ }^{7}$ it increases or decreases by $S_{\nu}(\mathfrak{X})$.

${ }^{6} R_{1}^{3}$ means the sheet of $\mathcal{R}^{3}$ in which $S_{1}(\mathfrak{X})$ is defined. (See p. 166). $S$ denotes the topological sum. $\cap$ means the intersection.

We suppose again that the point $\zeta=0$ lies neither inside $\zeta_{\nu}^{1}$ nor inside of ${ }^{1}$. Otherwise we must take into account the residue at $\mathcal{C}=0$ since the integrand of (2.1) can have a pole at this point.

${ }^{7}$ The surfaces $\mathcal{H}^{2}\left(2^{1}\right)$ are denoted as surfaces of separation of the representation (2.1) 


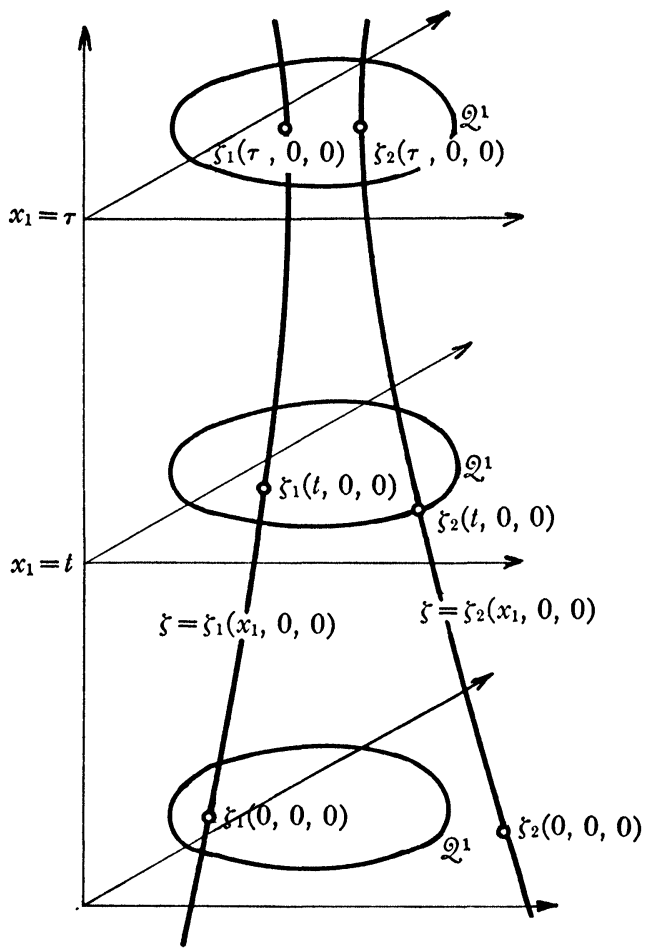

FIG. 1. The intersection of $Z^{3}$ with $\mathrm{E}\left[x_{2}=0, x_{3}=0\right]$

The curves $\mathcal{L}^{1}(\zeta)$ play also a role in another connection. Consider a closed curve $J^{1}$ in the schlicht $x_{1}, x_{2}, x_{3}$-space. To $J^{1}$ there corresponds by (2.6) a closed curve $\mathcal{F}^{1}(\zeta)$ in the $u$-plane. In the following we consider the case where the point $u=\phi(\zeta)$ lies inside of $\mathcal{F}^{1}(\zeta)$. If we construct the surface $\mathfrak{K}^{2}=\mathcal{S} \mathrm{z} \in \mathfrak{J}^{1}(\zeta) \mathcal{P}^{1}(\zeta, \mathrm{Z})$, then $\phi(\zeta)$ lies inside of $\mathcal{F}^{1}(\zeta)$ if and only if $\mathcal{F}^{2}$ cannot be reduced to a line without cutting $\mathcal{L}^{1}(\zeta)=\mathbb{P}^{1}(\zeta, \phi(\zeta))$. In general, this will happen if and only if the curve $\mathfrak{J}^{1}$ cannot be reduced to a point without cutting $\mathcal{L}^{1}(\zeta)$.

The statement that $J^{1}$ cannot be reduced to a point without cutting $\mathcal{L}^{1}(\zeta)$ we shall understand in the above sense.

The essential idea of the method of attack which will be used in the next section is the following:

Let $J^{1}$ be a closed curve in the $x_{1}, x_{2}, x_{3}$-space. If we divide it in an appropriate way, $J^{1}=S_{\nu} J_{\nu}^{1}$, then, by the operation $\mathrm{P}$, the differential $f(u, \zeta) d u$ will be transformed into $\Im_{\nu}(\mathfrak{X}) \cdot d \mathfrak{X}$, for $\mathfrak{X} \in \mathfrak{J}_{\nu}^{1}$, that is, we have $\left[\int_{\mathscr{2}^{1}} f(u, \zeta) d \zeta\right] d u=\mathfrak{S}_{\nu}(\mathfrak{X}) \cdot d \mathfrak{X}$. $\mathfrak{J}_{\nu}^{1}$ means that part of $\mathfrak{J}^{1}$ within whose points $f(u, \zeta)$ will be transformed into the branch $\mathfrak{S}_{\nu}(\mathfrak{X})$.

Here $\widetilde{S}_{\nu}(\mathfrak{X})$ is an appropriate branch of the algebraic harmonic 
vector $\mathfrak{S}(\mathfrak{X})$. (See (3.1).) Thus

$$
(1 / 2 \pi i) \int_{J^{1}}\left\{\int_{2^{1}}[f(u, \zeta) d \zeta]\right\} d u=\sum_{\nu} \int_{J^{1}} \mathfrak{S}_{\nu}(\mathfrak{X}) \cdot d \mathfrak{X}
$$

Changing the order of the integration in the left side member and applying the residue theorem for functions of one complex variable, we evaluate $(2.10)$.

3. Residue formula for line integrals. Let $J^{1}$ be a closed differentiable curve in the schlicht $x_{1}, x_{2}, x_{3}$-space. We place it in $R_{1}^{3}$ and suppose that $J^{1}$ intersects the cuts which bound $R_{1}^{3}$ at one (and only one) point $\&$. Thus $J^{1}$ is an open connected curve in $R_{1}^{3}$. Its end points (which coincide in the schlicht space with $\mathbb{E}$ ) are denoted by $\xi_{1}$ and $\mathbb{F}_{2}$.

Let $2^{1}$ be a closed curve in the $\zeta$-plane. We suppose (for simplicity's sake) that $\zeta_{1}\left(\xi_{1}\right)$ lies inside and $\zeta_{\nu}\left(\xi_{1}\right), \nu=2, \cdots, n$, lie outside of $2^{1}$. (See Figure 1.)

TheOREM. Let

$$
\begin{aligned}
\Im(\mathfrak{X}) & =\left[\frac{p(u, \zeta)}{\partial q(u, \zeta) / \partial \zeta}\right]\left[\mathfrak{i}_{1}+\frac{i\left(\zeta+\zeta^{-1}\right)}{2} i_{2}+\frac{\left(\zeta-\zeta^{-1}\right)}{2} \mathfrak{i}_{3}\right], \\
u & \equiv\left[x_{1}+(i / 2)\left(x_{2}+i x_{3}\right) \zeta^{-1}+(i / 2)\left(x_{2}-i x_{3}\right) \zeta\right]
\end{aligned}
$$

where $\zeta=\zeta\left(x_{1}, x_{2}, x_{3}\right)$ is a root of the equation

$$
q(u, \zeta) \equiv[u-\phi(\zeta)] \zeta^{s}=0,
$$

be a harmonic vector, the branches of which we denote by $\mathfrak{S}_{\nu}(\mathfrak{X})$, $\nu=1,2, \cdots, n$. The corresponding sheets of the multiply covered space of $\subseteq(\mathfrak{X})$ may be denoted by $\mathcal{R}_{\nu}^{3}$.

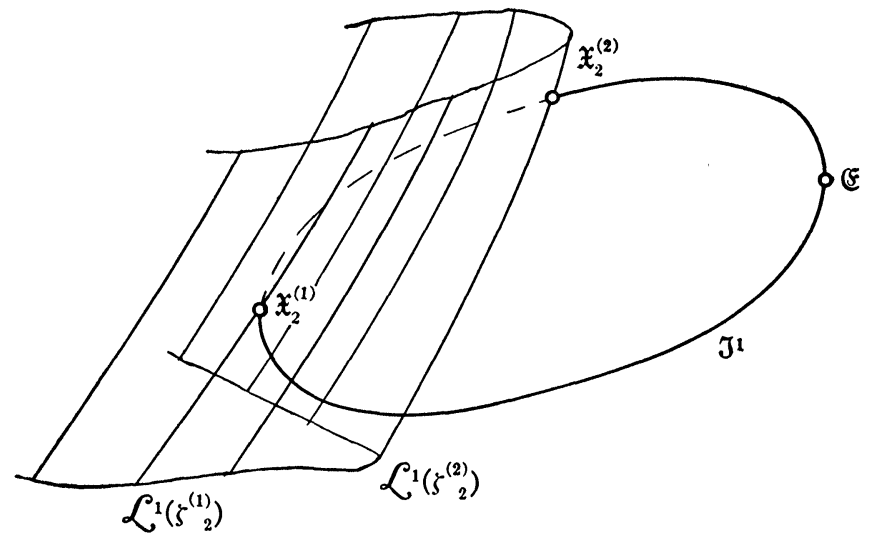

FIG. 2. The curve $\mathfrak{J}^{1}$ and the surface $\mathcal{H C}_{2}^{2}\left(2^{1}\right)$ 
Let $\mathfrak{J}^{1}$ and $2^{1}$ be curves in the $x_{1}, x_{2}, x_{3}$-space and in the $\zeta$-plane, respectively, with the above described properties.

Let finally $\left\{\mathfrak{X}_{\nu_{\mu}}^{(k)}\right\}, k=1,2 ; \mu=1,2, \cdots, p$ be the points of $\mathfrak{J}^{1}$ which satisfy the equation (3.2) for appropriate values ${ }^{9} \zeta=\zeta_{\nu}^{(k)}, \zeta_{\nu}^{(k)} \in 2^{1}$.

Then

$$
\int_{\mathfrak{E}_{1}}^{\mathfrak{E}_{2}} \mathfrak{\Im}_{1}(\mathfrak{X}) \cdot d \mathfrak{X}+\sum_{\mu=1}^{p} \int_{\mathfrak{X}_{\nu_{\mu}}^{(1)}}^{\mathfrak{X}_{\nu_{\mu}}^{(2)}} \mathfrak{S}_{\nu_{\mu}}(\mathfrak{X}) \cdot d \mathfrak{X}=2 \pi i \int_{S \mathcal{N}_{\mathrm{H}}^{1}} p[\phi(\zeta), \zeta] d \zeta
$$

where $\mathcal{N}_{\mathrm{H}}^{1}$ are the intervals of $2^{1}$, for which the filaments $\mathcal{L}^{1}(\zeta)$, of $\mathcal{S C}^{2}\left(2^{1}\right)$ lie inside of ${J^{1}}^{1} \zeta_{\nu_{\mu}}^{(k)}$ are their end points.

REMARK. We suppose here that the point $\zeta=0$ does not lie inside of $2^{1}$. Otherwise the components of the vector assume a form slightly different from that indicated in (3.1). These components in this case can be easily computed using the formulas of $[2, \S 2]$.

Proof. We consider the double integral

$$
I=\int_{J^{1}} \int_{2^{1}} \frac{p(u, \zeta) d \zeta d u}{q(u, \zeta)} .
$$

The integrand is an absolutely integrable function; we can replace the double integral by the iterated one and change the order of the integration. Thus,

$$
\begin{aligned}
I= & \int_{J^{1}}\left\{d x_{1} \int_{2^{1}} p q^{-1} d \zeta+d x_{2} \int_{2^{1}}(i / 2)\left(\zeta+\zeta^{-1}\right) p q^{-1} d \zeta\right. \\
& \left.+d x_{3} \int_{2^{1}}(1 / 2)\left(\zeta-\zeta^{-1}\right) p q^{-1} d \zeta\right\} .
\end{aligned}
$$

By (3.1), (2.4) and (1.2) we have $I=\int_{\mathfrak{I}^{1}} \mathfrak{B}\left(p q^{-1}, 2^{1}, \mathfrak{X}\right) \cdot d \mathfrak{X}$. According

\footnotetext{
${ }^{8}$ For simplicity's sake we suppose that $\mathfrak{J}^{1}$ intersects every $\mathcal{J}^{2}\left(2^{1}\right)$ in two points (if at all), and that it has no intersections with $\left.\mathcal{X}\right|_{1} ^{2}\left(2^{1}\right)$.

${ }^{9}$ The points $\mathfrak{X}$ of $\mathbb{R}_{1}^{3}$ which satisfy the equation (3.2) for a fixed $\zeta$ form the part of $\mathcal{L}^{1}(\zeta)$ which lies in $\mathbb{R}_{1}^{3}$. It consists of $n$ branches $\mathcal{L}_{p}^{1}(\zeta), p=1,2, \cdots, n$. The points $\mathfrak{X}$ of $R_{1}^{3}$ which satisfy (3.2) with $\zeta \in \mathcal{Q}^{1}$ form $n$ surfaces $\mathcal{O}_{p}^{2}\left(2^{1}\right)=\mathcal{S}_{\zeta \in \mathcal{Q}^{1}} \mathcal{L}_{p}^{1}(\zeta)$. (See p. 167.) The points $\left\{\mathcal{X}_{v_{\mu}}^{(k)}\right\}$ are the intersections of these surfaces with $\mathfrak{J}^{1}$.

The branch $\mathcal{X}_{\nu}^{2}\left(2^{1}\right)$ which corresponds to a given branch of $\mathfrak{S}(\mathfrak{X})$ can be determined as follows. For $\mathfrak{X}=\mathcal{E}_{1}$ we have $n$ roots of the equation $(3.2), \zeta=\zeta_{\nu}\left(\mathbb{E}_{1}\right), \nu=1$, $2, \cdots, n$. To every $\zeta_{\nu}\left(\xi_{1}\right)$ corresponds a uniquely determined branch $\Im_{\nu}(\mathfrak{X})$ of $\mathfrak{S}(\mathfrak{X})$. (See p. 166.) If the argument $\mathfrak{X}$ varies continuously in $\mathbb{R}_{1}^{3}, \zeta_{\nu}(\mathfrak{X})$ will move in the $\zeta$-plane. Let $\mathfrak{X}=\mathfrak{X}_{\tau}$ be a value such that $\zeta_{\nu}^{0}=\zeta_{\nu}\left(\mathfrak{X}_{\tau}\right) \in 2^{1}$. Since each $\mathcal{L}_{1}^{1}(\zeta)$ consists of $n$ disconnected branches the point $\mathfrak{X}_{r}$ lies in one of the lines $\mathcal{L}_{p}^{1}\left(\zeta_{\nu}^{0}\right)$. This branch of $\mathcal{L}_{1}^{1}\left(\zeta_{\nu}^{0}\right)$ corresponds to the branch $\Im_{\nu}(\mathfrak{X})$, and therefore $p=\nu$. The branch of $\mathcal{X}^{2}\left(\mathcal{Q}^{1)}\right.$ to which $\mathcal{L}_{\nu}^{1}\left(\zeta_{\nu}^{0}\right)$ belongs becomes $\mathcal{X}_{\nu}^{2}\left(Q^{1}\right)$.
} 
to the results on p. $167, \mathfrak{B}\left(p q^{-1}, 2^{1}, \mathfrak{X}\right)=\mathfrak{B}\left(p q^{-1}, 2^{1}, \mathfrak{E}_{1}\right)+\sum_{s=1}^{\mu} \mathfrak{S}_{v_{s}}(\mathfrak{X})$ for all points of $\mathfrak{J}^{1}$ which lie between $\mathfrak{X}_{\nu_{\mu}}^{(1)}$ and $\mathfrak{X}_{\nu_{\mu}}^{(2)}, \mu=1,2, \ldots$, p. Whence

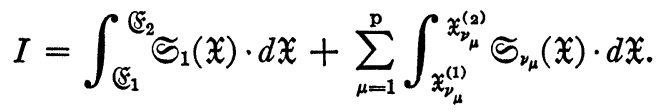

Changing the order of integration we obtain

$$
I=\int_{2^{1}} d \zeta\left[\int_{\mathcal{F}^{1}(\zeta)}[p(\tau, \zeta) / q(\tau, \zeta)] d \tau\right]=\int_{2^{1}} J(\zeta) d \zeta
$$

$\mathcal{F}^{1}(\zeta)$ is a closed curve in the complex $\tau$-plane and $q=[\tau-\phi(\zeta)] \zeta^{s}$. (See p. 167.) $J(\zeta)=2 \pi i p[\phi(\zeta), \zeta]$ or 0 if $\tau=\phi(\zeta)$ lies inside or outside of $\mathcal{F}^{1}(\zeta)$, respectively.

According to the considerations on p. $168, \phi(\zeta)$ lies inside of $\mathcal{F}^{1}(\zeta)$ if and only if $J^{1}$ cannot be reduced to a point without cutting $\mathcal{L}^{1}(\zeta)$. $I$ therefore is equal to the right-hand member of (3.3), which completes our proof.

EXAMPLE. We take $p=1, q=\zeta u, 2^{1}=\mathrm{E}\left[\zeta=e^{i t}, 0 \leqq t \leqq 2 \pi\right]$. The vector $\mathfrak{S}(\mathfrak{X})$ (see (3.1)) which we obtain in this case has two branches $\quad \Im_{1}(\mathfrak{X})=r^{-1}\left[\mathfrak{i}_{1}+x_{2}\left(x_{1}+r\right)^{-1} \mathfrak{i}_{2}+x_{3}\left(x_{1}+r\right)^{-1} \mathfrak{i}_{3}\right]$ and $\mathfrak{S}_{2}(\mathfrak{X})$ $=-r^{-1}\left[\mathfrak{i}_{1}+x_{2}\left(x_{1}-r\right)^{-1} \mathfrak{i}_{2}+x_{3}\left(x_{1}-r\right)^{-1} \mathfrak{i}_{3}\right], r^{2}=x_{1}^{2}+x_{2}^{2}+x_{3}^{2}$. The branches $\widetilde{S}_{1}$ and $\widetilde{S}_{2}$ are not connected in the real $x_{1}, x_{2}, x_{3}$-space. They are of a different form from (3.1) since $\zeta=0$ lies inside of $2^{1}$. We obtain $\mathfrak{S}_{1}(\mathfrak{X})$ and $\mathfrak{S}_{2}(\mathfrak{X})$ choosing the $x_{1}$ component of $\mathfrak{I}$ to be positive or negative, respectively. Further we have in this case:

$\mathcal{L}^{1}(\zeta)=\mathrm{E}\left[x_{1}=0, x_{2} \cos t+x_{3} \sin t=0\right]$ where $t=-i \log \zeta ; \mathcal{X}^{2}\left(2^{1}\right)=$ $\delta \mathcal{L}^{\prime}(\zeta)=\mathrm{E}\left[x_{1}=0\right]$.

Let $J^{1}=J_{1}^{1}+J_{2}^{1}$ be a closed curve in the $x_{1}, x_{2}, x_{3}$-space, where $J_{1}^{1}$ denotes the part which lies in $\mathrm{E}\left[x_{1}>0\right], \Im_{2}^{1}$ the part in $\mathrm{E}\left[x_{1}<0\right]$. By $\mathfrak{X}_{1}=\left(r_{1} \cos \phi_{1} \mathfrak{i}_{2}+r_{1} \sin \phi_{1} \mathfrak{t}_{3}\right)$ and $\mathfrak{X}_{2}=\left(r_{2} \cos \phi_{2} \mathfrak{i}_{2}+r_{2} \sin \phi_{2} \mathfrak{i}_{3}\right), 0<\phi_{1}<\pi / 2$, $\pi / 2<\phi_{2}<\pi$, we denote the intersections of $\mathfrak{J}^{1}$ with $\mathcal{X}^{2}\left(2^{1}\right)\left(=\mathrm{E}\left[x_{1}=0\right]\right)$. (We suppose that this intersection consists of two points.) Then

$$
\begin{aligned}
\sum_{\nu=1}^{2} \int_{\mathfrak{J}_{\nu}^{1}} \widetilde{S}_{\nu}(\mathfrak{X}) \cdot d \mathfrak{X} & =(1 / 2 \pi i) \int_{\mathcal{Q}^{1}} \zeta^{-1} d \zeta\left[\int_{\mathfrak{F}^{1}(\zeta)} u^{-1} d u\right] \\
= & \int_{\mathcal{N}_{1}^{1}}^{\zeta^{-1}} d \zeta-\int_{\mathcal{N}_{2}^{1}} \zeta^{-1} d \zeta,
\end{aligned}
$$

where $\mathcal{N}_{1}^{1}=\mathrm{E}\left[\zeta=e^{i t}, \phi_{1}+\pi / 2 \leqq t \leqq \phi_{2}+\pi / 2\right], \mathcal{N}_{2}^{1}=\mathrm{E}\left[\zeta=e^{i t}\right.$, $\left.\phi_{1}+3 \pi / 2 \leqq t \leqq \phi_{2}+3 \pi / 2\right]$.

REMARK 1. The generalization of the above considerations if $q$ is a polynomial of a higher degree than 1 presents no difficulties. (See [2].) 
We note further that our considerations can be generalized without essential difficulties to the case where $p$ and $q$ are entire functions.

RemarK 2 . We supposed that $2^{1}$ is a closed curve. However, our method can be applied also if $2^{1}$ is an open curve. An operator $\mathbf{P}\left(f, 2^{1}, \mathfrak{I}\right)$ with $2^{1}$ being an open curve transforms rational functions $f$ into harmonic functions with logarithmic singularities. The fact that the latter harmonic functions were not studied in detail prevented us from discussing this case here.

4. Residue formulas for surface integrals. Generalized Cauchy's formula. There exists another possibility of generalization of the residue formula, namely, in considering surface integrals.

Various relations for surface integrals can be obtained using Green's formula. In particular a well known formula for the value of the function in the domain in terms of boundary values of the function and its derivative can be established in this way.

In the case of two variables for a harmonic vector $\mathfrak{S}$ with curl $\mathfrak{S}=0$, $\operatorname{div} \mathfrak{S}=0, \mathfrak{S}(\mathfrak{X})$ may be expressed in terms of the boundary values of its components. (In Cauchy's integral formula for analytic functions which may be obtained in this way, the derivatives disappeared.)

It is clear that this procedure can be applied to harmonic vectors of several variables, provided that corresponding differential relations exist between the derivative of the components. ${ }^{10}$ As an illustration we shall derive a formula which can be considered as a generalization of Cauchy's formula.

Let $H(\mathfrak{X})$ be a harmonic function which is regular in a (schlicht) domain $\mathscr{D}^{3}$ with twice continuously differentiable boundary $\mathcal{B}^{2}$. Let $G_{\nu}$ be twice continuously differentiable functions, $\nu=1,2, \cdots, n$, such that

$$
\nabla H=\sum_{\nu=1}^{n} \nabla G_{\nu} \times a_{\nu}
$$

( $\times$ means vector product), where $\mathfrak{a}_{v}=a_{\nu 1} \mathfrak{i}_{1}+a_{v 2} \mathfrak{i}_{2}+a_{\nu 3} \mathfrak{i}_{3}$ are vectors with constant coefficients. Then

$$
H(\mathfrak{X})=(1 / 4 \pi) \iint_{\mathbb{B}^{2}}\left[H \nabla r^{-1}+\nabla r^{-1} \times \sum_{\nu=1}^{n} G_{\nu} \mathfrak{a}_{\nu}\right] \cdot d \mathfrak{D}
$$

where $d \mathfrak{0}=d x_{2} d x_{3} \dot{i}_{1}+d x_{1} d x_{3} \dot{i}_{2}+d x_{1} d x_{2} \dot{i}_{3}$.

${ }^{10}$ In particular this procedure has been used in the theory of functions of several complex variables to obtain generalizations of Cauchy's residue formula. See Bergman, Partial differential equations, Publication of Brown University, 1941 Supplementary Note III pp. SIII 8-SIII 9.

See also the further remarks concerning the other possibilities of generalizations of Cauchy's formulas mentioned there and Bergman, Revista de Ciencias, Lima, Peru, vol. 44 (1942) pp. 377-394 especially p. 391. 
Proof. By Green's formula,

$$
\begin{aligned}
H(\mathfrak{X}) & =(1 / 4 \pi)\left[\iint_{\mathcal{B}^{2}} H \nabla r^{-1} \cdot d \mathfrak{D}-\iint_{\mathcal{B}^{2}}{ }^{-1} \nabla H \cdot d \mathfrak{D}\right] \\
& =(1 / 4 \pi)\left(I_{1}-I_{2}\right) .
\end{aligned}
$$

By (4.1)

$$
\begin{aligned}
I_{2}= & \iint_{\mathcal{B}^{2}}\left[\frac{1}{r}\left(\frac{\partial H}{\partial x_{1}} d x_{2} d x_{3}+\frac{\partial H}{\partial x_{2}} d x_{1} d x_{3}+\frac{\partial H}{\partial x_{3}} d x_{1} d x_{2}\right)\right] \\
= & \iint_{\mathcal{B}^{2}} r^{-1}\left\{\sum_{\nu=1}^{n}\left(a_{3 \nu} \frac{\partial G_{\nu}}{\partial x_{2}}-a_{2 \nu} \frac{\partial G_{\nu}}{\partial x_{3}}\right) d x_{2} d x_{3}\right. \\
& +\sum_{\nu=1}^{n}\left(-a_{3 \nu} \frac{\partial G_{\nu}}{\partial x_{1}}+a_{1 \nu} \frac{\partial G_{\nu}}{\partial x_{3}}\right) d x_{1} d x_{3} \\
& \left.+\sum_{\nu=1}^{n}\left(a_{2 \nu} \frac{\partial G_{\nu}}{\partial x_{1}}-a_{1 \nu} \frac{\partial G_{\nu}}{\partial x_{2}}\right) d x_{1} d x_{2}\right\} .
\end{aligned}
$$

Integrating by parts we obtain

$$
I_{2}=-\iint_{\mathcal{B}^{2}}\left(\nabla r^{-1} \times \sum_{\nu=1}^{n} G_{\nu} \mathfrak{a}_{\nu}\right) \cdot d \mathfrak{0}
$$

since the line integrals cancel each other. Suppose now that we have a harmonic vector $\mathfrak{S}(\mathfrak{X})=\sum_{k=1}^{3} H^{(k)}(\mathfrak{X}) \dot{\mathfrak{t}}_{k}$, such that

$$
\nabla H^{(k)}=\sum_{\nu=1}^{3} \nabla H^{(\nu)} \times \mathfrak{a}_{\nu}^{(k)}, \quad k=1,2,3
$$

(4.2) yields

$$
\text { (4.4) } H^{(k)}(\mathfrak{X})=(1 / 4 \pi) \iint_{\mathcal{B}^{2}}\left[H^{(k)} \nabla r^{-1}+\nabla r^{-1} \times \sum_{\nu=1}^{3} H^{(\nu)} \mathfrak{a}_{\nu}^{(k)}\right] \cdot d \mathfrak{D} .
$$

Thus:

$$
\begin{aligned}
\mathfrak{S}(\mathfrak{X})=(1 / 4 \pi) \iint_{\mathcal{B}^{2}} & {\left[\mathfrak{S}\left(\nabla \boldsymbol{r}^{-1} \cdot d \mathfrak{D}\right)\right.} \\
& \left.+\sum_{k=1}^{3}\left[\left(\nabla r^{-1} \times \sum_{\nu=1}^{3} H^{(\nu)} \mathfrak{a}_{\nu}^{(k)}\right) \cdot d \mathfrak{v}\right] \mathfrak{t}_{k}\right] \\
= & (1 / 4 \pi) \iint_{\mathcal{B}^{2}}\left[\mathfrak{S}\left(\nabla r^{-1} \cdot d \mathfrak{D}\right)\right. \\
& \left.+\sum_{\nu=1}^{3} \sum_{k=1}^{3}\left[H^{(\nu)}\left(d \mathfrak{D} \times \nabla r^{-1}\right) \cdot \mathfrak{a}_{\nu}^{(k)}\right] \mathfrak{t}_{k}\right] .
\end{aligned}
$$


In particular if $\operatorname{div} \mathfrak{E}=0$ and curl $\mathfrak{S}=0$ then the relation (4.3) is satisfied, and we can represent $\mathfrak{S}(\mathfrak{X})$ in the above form (4.4).11

REMARK. $d \mathfrak{0}=\mathfrak{n}_{1} d o$, where $\mathfrak{n}_{1}$ is the unit vector in the direction of the interior normal, and $d o$ the area of the surface element. (We note that $d x_{m} d x_{n}$ means $+\left|d x_{m} d x_{n}\right|$ or $-\left|d x_{m} d x_{n}\right|$, the sign being chosen in such a manner that the above relation for $d_{0}$ is valid.)

(4.2) can also be obtained using the Stokes' formula. In fact

$$
I_{2}=\sum_{\nu} \iint_{\mathcal{B}^{2}} \mathfrak{n}_{1} r^{-1}\left(\nabla G_{\nu} \times \mathfrak{a}_{\nu}\right) d o=\sum_{\nu} \iint_{\mathcal{B}^{2}} \mathfrak{n}_{1} r^{-1}\left(\nabla \times G_{\nu} \mathfrak{a}_{\nu}\right) d o
$$

since the $a_{k v}$ are constants. By Stokes' formula

$$
\begin{aligned}
\iint_{\mathcal{B}^{2}} \mathfrak{n}_{1}\left(\nabla \times r^{-1} G_{\nu} \mathfrak{a}_{\nu}\right) d o= & \iint_{\mathcal{B}^{2}} \mathfrak{n}_{1} r^{-1}\left(\nabla \times G_{\nu} \mathfrak{a}_{\nu}\right) d o \\
& +\iint_{\mathcal{B}^{2}} \mathfrak{n}_{1} G_{\nu}\left(\nabla \times r^{-1} \mathfrak{a}_{\nu}\right) d o=0,
\end{aligned}
$$

since $\mathbb{B}^{2}$ is a closed surface. Therefore

$$
I_{2}=-\sum_{\nu} \iint_{\mathcal{B}^{2}} \mathfrak{n}_{1} G_{\nu}\left(\nabla \times r^{-1} \mathfrak{a}_{\nu}\right) d o .
$$

We also note that formulas expressing the pressure on an immersed body (analogous to those of the two-dimensional theory) can be obtained using the fact that $\iint_{\mathcal{B}^{2}}\left[\mathfrak{n}_{1}|\mathfrak{S}|^{2}-2 \mathfrak{S}\left(\mathfrak{n}_{1} \mathfrak{S}\right)\right] d o$ does not change when $\mathscr{B}^{2}$ is deformed, if curl $\mathfrak{E}=\operatorname{div} \mathfrak{S}=0$.

REMARK. The method presented here can obviously be applied if the components $H^{(n)}$ of a vector $\mathfrak{S}(\mathfrak{X})$ are connected by the relations

$$
\sum_{n=1}^{3} \mathfrak{a}_{k n} \cdot \nabla H^{(n)}+\mathfrak{b}_{k} \cdot \mathfrak{g}+C_{k}=0, \quad k=1,2,3,
$$

where $\mathfrak{a}_{k n}=\left(a_{k n}^{(1)} \mathfrak{i}_{1}+a_{k n}^{(2)} \mathfrak{i}_{2}+a_{k n}^{(3)} \mathfrak{i}_{3}\right), \quad \mathfrak{b}_{k}=\left(b_{\mathfrak{k}}^{(1)} \mathfrak{t}_{1}+b_{k}^{(2)} \mathfrak{i}_{2}+b_{k}^{(3)} \mathfrak{t}_{3}\right)$, and the . determinants $a_{k n}^{(s)} \neq 0$, for $n=1,2,3$.

Analogous formulas are also valid if $H^{(k)}$ satisfy certain elliptic differential equations and if we introduce instead of $r^{-1}$ a fundamental solution of this equation.

\section{BIBLIOGRAPHY}

1. S. Bergman, Math. Zeit. vol. 24 (1926) pp. 641-669.

2. - Math. Ann. vol. 99 (1928) pp. 629-658.

BROWN UNIVERSITY

${ }^{11}$ In the case where curl $\mathfrak{S}=\operatorname{div} \mathfrak{S}=0$ similar formulas were given by von Mises (an address presented at the New York meeting of the Society on April 4, 1942, by invitation of the Program Committee) and by Fulton and Rainich (Amer. J. Math. vol. 54 (1932) pp. 235-241). 